\title{
Detection of High Risk Human Papillomavirus Cervical Infections by the Hybrid Capture in Asunción, Paraguay
}

Laura Mendoza Torres, Malvina Páez, Ariel Insaurralde, María Isabel Rodriguez, Amalia Castro and Elena Kasamatsu Deparment of Public Health and Epidemiology, Research Institute of Health Science, University of Asunción; Asunción, Paraguay

Cervical cancer is the most frequent malignant tumour of women in Latin America being human papillomavirus (HPV) the main cause. The aim of this study was to increase the knowledge about the cervical infections with oncogenic HPV types (HR-HPV) in Asuncion, Paraguay. Two hundred and seventy-two cervical samples were analyzed using hybrid capture II assay (HCA II) for HR-HPV. The frequency of HR-HPV in the study group was $44 \%$. HR-HPV was detected in $25 \%$ of the women negative for squamous intraepithelial lesions (NSIL), $72 \%$ with atypical squamous cells of undetermined significance (ASCUS), $68 \%$ with low SIL and $78 \%$ with high SIL. A moderate concordance was observed between HCA II assay and cytology (kappa: 0.43 IC $_{95 \% 0.3-0.5}$ ). It was detected a high frequency of HR-HPV in women from 11 to 30 years old and in those over 60 years old. The data obtained in this study showed a high frequency of HR-HPV in woman with NSIL and ASCUS, which corroborate that the use of cytology together with HCA II assay for HR-HPV could improve remarkably the efficiency of screening programs of cervical cancer in Paraguay. Furthermore, these findings point out the need for the periodical follow-up of HR-HPV infections in older women.

Key-Words: High risk human papillomavirus, hybrid capture II assay, cytology.

Cervical cancer is the most frequent malignant neoplasia in the female population of Latin American countries, among these, Paraguay occupies the third place in incidence with a rate of 53.2 x 100,000 women, only surpassed by Haiti and Bolivia [1,2].

Human Papillomavirus (HPV) is the most prevalent virus involved in sexually-transmitted diseases worldwide, being an important public health challenge. HPV is also considered the main cause of cervical cancer and squamous intraepithelial lesions (SIL) [3,4].

Approximately 40 distinct HPV types are known to infect the anogenital mucosa and they are grouped commonly into high risk (HR-HPV) and low risk (LR-HPV) categories on the basis of known epidemiological association with preneoplasic/ neoplasic and benign lesions, respectively [5]. HR-HPV are detected in more than $95 \%$ of the cervical carcinoma cases [6,7].

Diagnosis of cervical disease, determined by the presence of abnormal cervical epithelial cells, is usually obtained by microscopic examination of Papanicolaou-stained smears (PAP). This is a simple and cheap method that allows detecting effects caused by HPV infection that consists in the observation of coilocitic cells, but does not permit to determine the presence of HR-HPV infections [8].

HPV could not be isolated in cells, therefore the diagnosis test depends on molecular techniques. The hybrid capture II assay (HCA II) is the only molecular technique approved by the Food and Drugs Administration (FDA) designed to detect 13 types of HR-HPV and 5 of LR-HPV, even in apparently

Received on 18 April 2009; revised 18 June 2009.

Address for correspondence: Dr. Laura Torres. Iturbe 1184, P.O. Box 2017, Asuncion, Paraguay. Tel.: 59521333 549; fax: 59521445184. E-mail address: msclauramt@yahoo.com.br (Mendoza Torres, L).

The Brazilian Journal of Infectious Diseases 2009;13(3):203-206. (C) 2009 by The Brazilian Journal of Infectious Diseases and Contexto Publishing. All rights reserved. normal tissues, due to the high sensitivity that permits the detection of $1 \mathrm{pg} / \mathrm{mL}$ of viral DNA [9].

In Paraguay, a country with a high incidence of cervical cancer, there are only two studies related to HPV typing in female genital invasive cancer by using Polymerase Chain Reaction (PCR) $[10,11]$. Due to the fact that the HCA II assay was recently introduced in our country, the goal of the present study was to determine the frequency of HR-HPV in cervical samples of women, in order to contribute to a better knowledge on HR-HPV cervical infections in Asuncion, capital city of Paraguay, with a population of 520,000 inhabitants. The findings were correlated with cytological diagnosis and age.

\section{Material and Methods}

Study Population

The population under study included 272 women which were attended at the HPV Laboratory that belongs to the Instituto de Investigaciones en Ciencias de la Salud from Universidad Nacional de Asunción, Paraguay, from May 2006 to December 2007.

The participants were included following a consecutive order by using the selection criteria previously established such as a previous cytological diagnosis realized by the specialized physician and without histerectomy. The results of cervical cytology were classified using the Bethesda System, 2001 [12]. All the data were processed respecting the confidentiality of the participants.

Detection of HR-HPV by Hybrid Capture II Test

The smears were collected using a cervical cytobrush and then transported in Digene Specimen Transport Medium (Digene Diag, Md).

The kit detects HR-HPV types 16, 18, 31, 33, 35, 39, 45, 51, 52, 56, 58, 59 and 68. Following the manufacturer's protocol, the specimens were treated with sodium hydroxide in order to hydrolyze specimen RNA and to denature the DNA. The 
liberated single-strand DNA was hybridized in solution with an RNA probe mix consisting of high risk HPV types. Each reaction mixture, containing any RNA-DNA hybrids, was transferred to a capture tube sensitized with antibodies antihybrids, immobilizing them. The bound RNA-DNA hybrids were then captured by an alkaline phosphatase-conjugated antibody against the hybrids. Unbound material was removed by washing, and a dioxetane-based chemiluminescent reagent, Lumi-Phos 530 was added as a substrate for alkaline phosphatase. The luminiscence produced by the reaction was measured by a luminometer. Light measurements were expressed as relative light units (RLUs). As a negative control, sonicated herring sperm DNA in Digene transporting medium (100 mg/mL) was used. Triplicate specimens of HPV 16 DNAs at $1.0 \mathrm{pg} / \mathrm{mL}$ were used as the positive controls. The RLU measurements for each specimen were divided by the RLU average of the three positive controls (PCs) to give a ratio of specimen RLU/PC. A ratio of 1.0 or greater was considered as positive for HR-HPV genome.

\section{Statistical Analysis}

The statistical analysis was realized using a descriptive statistical software (EpiInfo version 3.2).

The concordance between the results of HCA II for HRHVP and cytological diagnosis was realized using the kappa index with a interval of confidence of 95\%, indicating that values under 0.20 mean a poor concordance, 0.20 to 0.40 a weak concordance, 0.41 to 0.60 a moderate concordance, 0.61 to 0.80 a good concordance and over 0.80 to 1 a very good concordance [13].

\section{Results}

According to the cytological diagnosis, 58\% (159/272) of the women presented a result negative to squamous intraepithelial lesions (NSIL), 9\% (25/272) presented atypical squamous cells of undetermined significance (ASCUS), $29 \%$ (79/272) presented low grade squamous intraepithelial lesions (LSIL) and 3\% (9/272) presented high grade squamous intraepithelial lesions in situ carcinoma (HSIL).

The frequency of HR-HPV detected by HCA II assay in the group of study was 44\% (119/272 women). Comparing to cytological diagnosis, HR-HPV was detected in $25 \%$ of the women with NSIL, 72\% with ASCUS, 68\% with LSIL and 78\% with HSIL. The frequency of HR-HPV by HCA II according to the cytological diagnosis is shown in Table 1.

A moderate concordance was observed between the results of HCA II assay and cytology of women with NSIL and SIL with a kappa index value of $0.43\left(\mathrm{IC}_{95 \% 0.3-0.5}\right)$. The HR$\mathrm{HPV}$ results of 25 women with unspecific cytological diagnosis of ASCUS were excluded to determine the concordance of the tests. The results for HR-HPV by HCA II assay of 247 women with SIL and NSIL are shown in Table 2.

The average age was $34 \pm 10$ years old (from 17 to 65 years old). A high frequency of HR-HPV was detected in women in the range of 11 to 30 years old and over 60 years old; with a notorious incidence in women under 21 years old (75\%). Positive results of HR-HPV by HCA II assay by age ranges are shown in Table 3.

\section{Discussion}

The World Health Organization reported that cervical cancer is the second cause of malignant neoplasia and death in women worldwide [4]. Epidemiological studies have demonstrated that HR-HPV is the main cause of this disease. However, it is necessary to use complementary diagnosis tools that allow a detection of HR-HPV genome, in order to increase the performance of conventional morphologic diagnostic methods usually used for screening cervical cancer [3].

In this study, cervical samples were analyzed using the HCA II assay in order to detect the HR-HPV genome. Viral infections were observed in $44 \%$ of the women. This finding agrees with previous studies where a comparable population and methodology of analysis was used [4,14]. Epidemiological studies showed that almost $50 \%$ of sexually active women between 15 and 49 years old are infected by HPV, becoming a worldwide public health problem [4,14].

A moderate concordance was observed between HCA II assay and cytology (kappa: $0.43 \mathrm{IC}_{95 \% 0.3-0.5}$ ). 25\% of the women with N-SIL were positive to HR-HPV. This could be due to the high sensitivity of almost $100 \%$ by the HCA II assay, that allowed detecting the HR-HPV infection even in apparently normal tissues and revealing silent infections that may be responsible for the maintenance of HPV infections in the general population $[8,15,16]$. Previously, Koutsky et al. have described women with no cytological abnormalities presenting positive results for HPV genome, and they have also observed that $30 \%$ of the women analyzed developed CIN within the next two years, suggesting that there is a higher risk for these women for developing CIN [17].

Thirty-two percent and 22\% of women with LSIL and HSIL, respectively, were negative for HR-HPV. These contrasting results could be due to a lower copy number of the HR-HPV genome infection; untested types of HPV HCA II assay or unidentified reasons, such as DNA testing errors [18,14].

Some of those conflicting cases between cytology and HR-HPV genome detection could be due to a mistake in the cytological diagnosis assay, which presented almost $20 \%$ of false negative results (lower sensitivity) and 15\% of false positive results (lower specificity) [18,14].

Seventy-five percent of women with ASCUS were positive for HR-HPV genome. Previous studies have demonstrated the efficacy of HCA II assay in the orientation of unspecific cytological diagnosis of ASCUS, suggesting that a positive result for HR-HPV genome means a possible presence of SIL [15,19-21].

Regarding the age ranges, a high frequency of positive cases of HR-HPV was observed in women from 11 to 30 years old, mainly in women under 21 years old. This high percentage of infected women could be due to a young cervical tissue, with 
Table 1. Positive results of high risk HPV (HR-HPV) by hybrid capture II assay (HCA II) according to the cytological diagnosis of women.

\begin{tabular}{lcc}
\hline Cytological diagnosis & Number of women & HR-HPV infection \\
\hline & & $\mathbf{n}(\%)$ \\
NSIL & 159 & $40(25)$ \\
ASCUS & 25 & $18(72)$ \\
LSIL & 79 & $54(68)$ \\
HSL & 9 & $7(78)$ \\
Total & 272 & $119(44)$ \\
\hline
\end{tabular}

NSIL: negative to squamous intraepithelial lesions. ASCUS: atypical squamous cells of undetermined significance. LSIL: low grade squamous intraepithelial lesions. HSIL: high grade squamous intraepithelial lesions/ in situ carcinoma.

Table 2. Results of high risk HPV by hybrid capture II assay (HCA II) from women with and without squamous intraepithelial lesions.

\begin{tabular}{lccc}
\hline Cytological diagnosis & \multicolumn{2}{c}{ HR-HPV infection } & Total \\
\cline { 2 - 3 } & Positive N (\%) & Negative N (\%) & N \\
\hline SIL & $61(69)$ & $27(31)$ & 88 \\
NSIL & $40(25)$ & $119(75)$ & 159 \\
Total & $101(41)$ & $146(59)$ & 247 \\
\hline
\end{tabular}

SIL: squamous intraepithelial lesions. NSIL: negative for squamous intraepithelial lesions. Kappa 0.43 ( IC $_{95 \%} 0.3-0.5$ ).

Table 3. Positive results for high risk HPV (HR-HPV) by hybrid capture II (HCA II) assay according to the age of the women (in years).

\begin{tabular}{lcc}
\hline Age range & Number of women & $\begin{array}{c}\text { HR-HPV infection } \\
\mathbf{n}(\%)\end{array}$ \\
\hline $11-20$ & 12 & $9(75)$ \\
$21-30$ & 107 & $62(58)$ \\
$31-40$ & 79 & $21(27)$ \\
$41-50$ & 53 & $19(36)$ \\
$51-60$ & 13 & $5(38)$ \\
$61-70$ & 3 & $2(67)$ \\
Total & $267 *$ & $118(44)$ \\
\hline
\end{tabular}

*Age was not determinate in 5/272 women included in the present study.

probably a higher transformation activity; making them more vulnerable to sexual transmitted diseases, such as HPV [4].

In women from 31 to 60 years old it was observed a decrease of positive cases by HR-HPV infection. This finding agrees with previous studies that showed a prevalence of HPV infection of almost $30 \%$ in women under 30 years old, which decreases to $10 \%$ between 30 and 50 years old [22].

The decrease in the rate of infection observed with the increase in age can be explained because from $80 \%$ to $90 \%$ of women less than 30 years old were already exposed to any HPV infection, developed a natural immunity and could eliminate the infection in a period of following 24 months. It is also interesting to note that the remaining $10 \%$ to $20 \%$ of women over this age that are persistent infected by HPV have a great potential of developing SIL if they are not correctly diagnosed and treated [22].

Hence, several authors concluded that the use of cytological diagnosis complemented with DNA test for detection of HR-HPV genome, helps to increase the efficiency of screening programs allowing the early detection of cervical lesions at age intervals over 30 years old [17].

Interestingly, it was observed an increase of HR-HPV infections in women over 60 years old (67\%). This finding also agrees with previous studies realized in other Latin American countries that described an increase in the rate of infection caused by both HR and low risk HPV (LR-HPV) $[23,24]$. This fact could be due to a new exposition to HPV through a sexual partner; a decrease of the immune system associated to the age that results in an increase of the reactivation of latent infections or to other factors [24].

Summing up, the results obtained in this study corroborate that the use of cytology together with the detection of HRHPV by HCA II assay could improve remarkably the efficiency of screening programs of cervical cancer in Paraguayan women over 30 years old, allowing the identification of a group of women with high risk of developing SIL and supporting the 
necessity of follow-up by screening with the HPV test in older women groups.

\section{Acknowledgements}

The authors are grateful to the scientific and ethic workers' committee of the Instituto de Investigaciones en Ciencias de la Salud from Universidad Nacional de Asunción, Paraguay and especially to Eva Nara, $\mathrm{PhD}$ for the critical review of this study. We also thank to the specialized physician for collaborating with this study.

\section{References}

1. Parkin D.M., Bray F., Ferlay J., Pisan P. Global cancer statistics 2002. C A Cancer J Clin 2005;55:74-108.

2. Lewis Merle J. Análisis de la Situación del cáncer cervicouterino en América Latina y el Caribe. Organización Panamericana de la Salud/ Organización Mundial de la Salud (OPS/OMS). Washington DC, 2004.

3. Muñoz N., Bosch F.X., de Sanjose S. et al. Epidemiologic classification of human papillomavirus types associated with cervical cancer. N Engl J Med 2003;348(6):518-27.

4. Carestiato F.N., Silva K.S., Dimetz T., et al. Prevalence of Human Papillomavirus Infection in the Genital Tract Determined by Hybrid Capture Assay. Braz J Infect Dis 2006;10(5):331-6.

5. zur Hausen H. Papillomavirus infections-a major cause of human cancers. Biochim Biophys Acta 1996;1288(2):55-78.

6. Walboomers J.M., Jacobs M.V., Manos M.M. et al. Human papillomavirus is a necessary cause of invasive cervical cancer worldwide. J Pathol 1999;189(1):12-9.

7. Wright, Jr. T.C., Schiffman M., Solomon D. et al. Interim guidance for the use of human papillomavirus DNA testing as an adjunct to cervical cytology for screening. Obstet Gynecol 2004;103(2):304-9.

8. Dôres G.B., Taromaru E.K., Bonomi C.G. et al. HPV infection detected by hybrid capture II: correlation with morphological findings. DST - J Bras Doenças Sex Transm 2005;17(4):255-8.

9. Carvalho M.O.O., Almeida R.W., Leite F.M.S. Detection of Human Papillomavirus DNA by the Hybrid Capture Assay. Braz J Infect Dis 2003;7(2):121-5.

10. Kasamatsu E., Ascurra M., Hullin C., Nakajima T., Shozawa T. Detection and typing of human papillomavirus DNA in invasive squamous cell carcinoma of the cervix by polymerase chain reaction and its correlation with histopathological findings. Anales de la Facultad de Ciencias Médicas 1996;29(1-2):317-33.
11. Rolon P.A., Smith J.S., Muñoz N. et al. Human papillomavirus infection and invasive cervical cancer in Paraguay. Int J Cancer 2000;85(4):486-91.

12. Solomon D., Davey D., Kurman R. et al. The 2001 Bethesda System: terminology for reporting results of cervical cytology. JAMA. 2002;287(16):2114-9.

13. Altman D.G. Practical statistics for medical research. New York: Chapman and Hall; 1991.

14. Carvalho M.O.O., Carestiato F.N., Perdigao P.H. Human papillomavirus infection in Rio de Janeiro, Brazil: a retrospective study. Braz J Infect Dis 2005;9(5):398-404.

15. Lorincz A.T., Richart R.M. Human papillomavirus DNA testing as an adjunct to cytology in cervical screening programs. Arch Pathol Lab Med 2003;127(8):959-68.

16. Castle P.E., Wacholder S., Sherman M.E. et al. Absolute risk of a subsequent abnormal pap among oncogenic human papillomavirus DNA-positive, cytologically negative women. Cancer 2002;95(10):2145-51.

17. Koutsky L.A., Holmes K.K., Critchlow C.W. et al. A cohort study of the risk of cervical neoplasia grade 2 or 3 in relation to papillomavirus infection. N Engl J Med 1992;327:1272-8.

18. Morrison H. Human papillomavirus absence predicts normal cervical histopathologic findings with abnormal papanicolaou smears. J Hum Virol 1993;4:283-7.

19. Solomon D., Schiffman M., Tarone R. Comparison of three management strategies for patients with atypical squamous cells of undetermined significance: Baseline results from a randomized trial. J Natl Cancer Inst 2001;93:293-9.

20. Ferreccio C., Bratti M.C., Sherman M.E. et al. A comparison of single combined visual cytologic, and virologic tests as screening strategies in region at high risk of cervical cancer. Cancer Epidemiol Biomarkers Prev 2003;12(9):815-23.

21. Arbyn M., Paraskevaidis E., Martin-Hirsch P. et al. Clinical utility of HPV DNA detection: triage of minor cervical lesions, follow - up of women treated for high-grade CIN. An update of pooled evidence. Gynecol Oncol 2005;99(Suppl 3):7-11.

22. Nazzal O.N., Suárez E.P., Larraguibel R.P. et al. Lesiones preinvasoras de cuello uterino: una visión actual. Rev Chil Obstet Ginecol 2006;71(5):341-8.

23. Ferreccio C., Prado R.B., Luzoro A.V. et al. Population-based prevalence and age distribution of human papillomavirus among women in Santiago, Chile. Cancer Epidemiol Biomarkers Prev 2004;13(12):2271-6.

24. Castle P.E., Schiffman M., Herrero R. et al. A prospective study of age trends in cervical human papillomavirus acquisition and persistence in Guanacaste, Costa Rica. J Infect Dis 2005;191(11):1808-16. 